\title{
Micromagnetic Simulations of Magnetic Particles Embedded in Magnetic or Non-Magnetic Matrices ${ }^{\dagger}$
}

\author{
Devika Sudsom and Andrea Ehrmann *
}

Citation: Sudsom, D.; Ehrmann, A. Micromagnetic Simulations of Magnetic Particles Embedded in Magnetic or Non-Magnetic Matrices. Mater. Proc. 2021, 4, 80. https:// doi.org/10.3390/IOCN2020-07940

Academic Editors: Ana María Díez-Pascual, Antonio Di Bartolomeo and Guanying Chen

Published: 11 November 2020

Publisher's Note: MDPI stays neutral with regard to jurisdictional claims in published maps and institutional affiliations.

Copyright: (c) 2020 by the authors. Licensee MDPI, Basel, Switzerland. This article is an open access article distributed under the terms and conditions of the Creative Commons Attribution (CC BY) license (http://creativecommons.org/licenses /by/4.0/).
Faculty of Engineering and Mathematics, Bielefeld University of Applied Sciences, 33619 Bielefeld, Germany; devika.sudsom@fh-bielefeld.de

* Correspondence: andrea.ehrmann@fh-bielefeld.de

† Presented at the 2nd International Online-Conference on Nanomaterials, 15-30 November 2020; Available online: https://iocn2020.sciforum.net/.

\begin{abstract}
Combining soft and hard magnetic materials is not only of technological importance in diverse spintronics elements, but also of high interest in basic research. Here, we report on different arrays combining iron and nickel, e.g., by embedding circular nanodots of one material in a matrix of the other. Micromagnetic simulations were performed using OOMMF. Our results show that magnetization reversal processes are strongly influenced by neighboring nanodots and the magnetic matrix in which the nanodots are embedded, respectively, which becomes macroscopically visible by several steps along the slopes of the hysteresis loops. Such material combinations allow for preparing quaternary memories and are thus highly relevant for applications in data storage and processing.
\end{abstract}

Keywords: micromagnetic simulation; OOMMF; nanodots; antidots; array; spintronics

\section{Introduction}

The magnetic properties and magnetization reversal of single nanoparticles or nanoparticle arrays are defined by the dimensions and shapes of the nanoparticles as well as by their material. This interplay between magneto-crystalline and shape anisotropy can lead to unexpected and technologically applicable effects [1,2]. In particular, round and square nanodots, nanodot arrays and antidot arrays are often examined since they allow for building vortices with reduced in-plane stray fields [3-8].

On the other hand, combining different materials either in the form of thin layer stacks or in one plane offers new possibilities of controlling magnetization reversal processes. Most recently, Salaheldeen et al. investigated the overall anisotropies in magnetic hard/soft bilayer antidot arrays and found a crossover from magnetic in-plane to out-of-plane anisotropy due to the interface exchange coupling between the hard Co and the soft Py layer [9]. Verba et al. also coupled soft ferromagnetic thin films and nanodots to a hard magnetic antidot matrix, in this way allowing for tuning the helicity of a vortex of skyrmion in this material [10]. Negusse and Williams reported on exchange-spring materials, based on combining soft and hard magnetic layers [11]. Kim et al. prepared soft/hard magnetic composite fibers by electrospinning, combining iron oxide (soft) and cobalt ferrite (hard) magnetic materials, and found a higher saturation magnetization and coercivity than in pure $\mathrm{CoFe}_{2} \mathrm{O}_{4}$ [12]. Similarly, Zhang et al. prepared $\mathrm{CoFe}_{2} \mathrm{O}_{4} / \mathrm{La}_{0.7 \mathrm{Sr}}{ }_{3} \mathrm{MnO}_{3}$ soft/hard composites and found different saturation magnetization for different mixing ratios [13].

In our group, previously different in-plane combinations of hard/soft magnetic materials were examined, such as bow-tie structures, double-wedges and semi-squares $[14,15]$. Combining the approaches of nanodot/antidot arrays and soft/hard magnetic materials, here, we report, to the best of our knowledge for the first time, on magnetically 
hard nanodots embedded in a magnetically soft matrix and vice versa and compare both situations with a pure magnetic hard nanodot array as well as with a mixed array. Our results show clearly different coercive fields and hysteresis loop shapes due to combining both magnetic materials in a nanodot/antidot structure.

\section{Materials and Methods}

Micromagnetic simulations were performed using the micromagnetic simulator OOMMF (object-oriented micromagnetic framework) [16] by dynamically solving the Landau-Lifshitz-Gilbert equation dynamically on a mesh built by finite differences [17]. To prepare bi-material maps, the function ImageAtlas was used, defining material positions by image files. Material parameters for iron (Fe) and nickel (Ni) were chosen in agreement with average literature values: magnetization at saturation $\mathrm{Ms}, \mathrm{Fe}=1700 \times 10^{3}$ $\mathrm{A} / \mathrm{m}\left(\mathrm{Ms}, \mathrm{Ni}=490 \times 10^{3} \mathrm{~A} / \mathrm{m}\right)$, exchange constant $A_{\mathrm{Fe}}=21 \times 10^{-12} \mathrm{~J} / \mathrm{m}\left(\mathrm{ANi}_{\mathrm{Ni}}=9 \times 10^{-12} \mathrm{~J} / \mathrm{m}\right)$, magneto-crystalline anisotropy constant $\mathrm{K}_{1, \mathrm{Fe}}=48 \times 10^{3} \mathrm{~J} / \mathrm{m}^{3}\left(\mathrm{~K}_{1, \mathrm{Ni}}=-5.7 \times 10^{3} \mathrm{~J} / \mathrm{m}^{3}\right)$. Average values between both materials were used to define the borders [18]. To take into account the common production methods of such nanostructures by sputtering, arbitrary crystallographic orientations are chosen in each cubic grain of diameter $3 \mathrm{~nm}$. Setting the Gilbert damping constant to $\alpha=0.5$ results in the simulation of a quasistatic case. Simulations were performed by sweeping the external magnetic field from positive to negative saturation and back again. Investigating the transverse magnetization components $\mathrm{M}_{\mathrm{T}}$ in addition to the longitudinal hysteresis loops $M_{L}$ ensures avoiding minor loops. Here, the results of field sweeps along $45^{\circ}$ (cf. Figure 1) are shown; future simulations will investigate further field orientations.

The nanodot areas under examination are sketched in Figure 1. Four magnetic dots of diameter $105 \mathrm{~nm}$ are embedded in a square of side length $210 \mathrm{~nm}$. Outside the dots, there is either air or the second magnetic material, in this way forming an antidot array. The system height is $15 \mathrm{~nm}$.
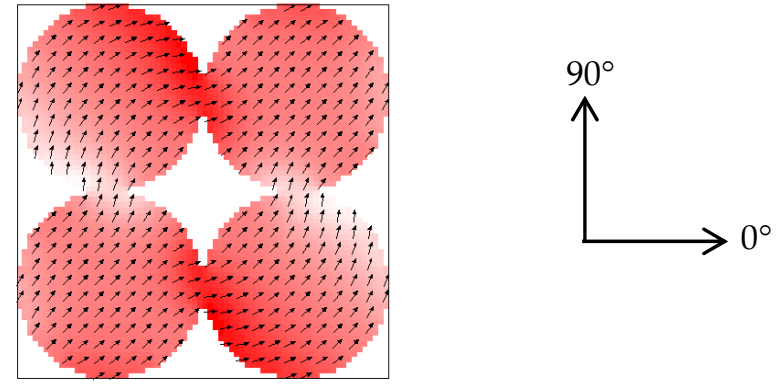

Figure 1. Sketch of the nanodot arrays under examination consisting of four touching nanodots (diameter $105 \mathrm{~nm}$ ), embedded in air or in the other magnetic material, as well as orientation of the external magnetic field angles.

\section{Results and Discussion}

To start with a common structure, Figure 2 depicts longitudinal and transverse hysteresis loops $\mathrm{ML}_{\mathrm{L}}$ and $\mathrm{M}_{\mathrm{T}}$, respectively, as well as snapshots during magnetization reversal from positive to negative saturation of the system consisting of four iron nanodots in air. 


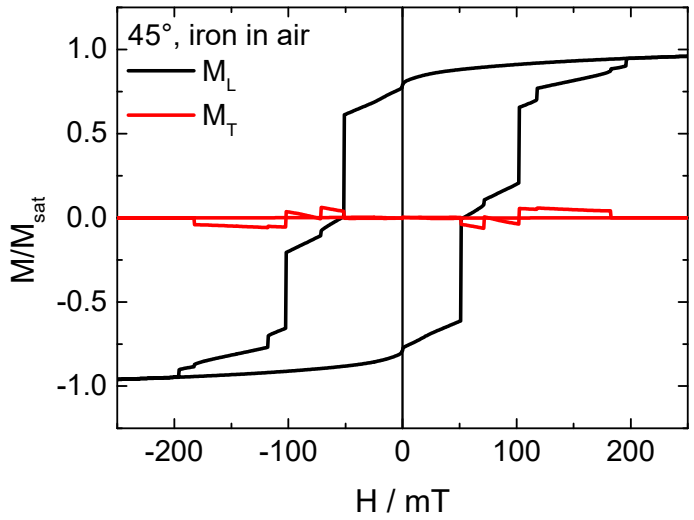

(a)
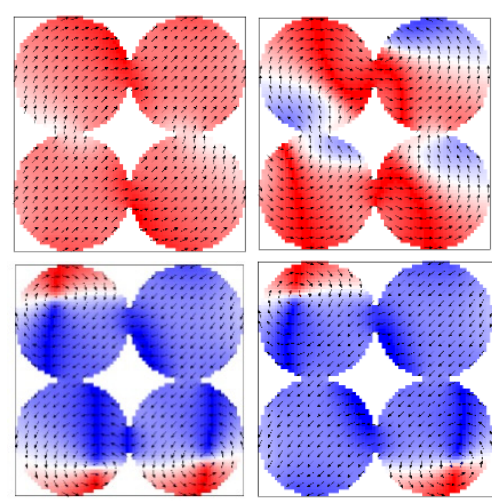

(b)

Figure 2. (a) Hysteresis loop and (b) snapshots of magnetization reversal from positive to negative saturation for a pure iron nanodot array in air.

As Figure $2 \mathrm{~b}$ clearly shows, even in this apparently simple system, magnetization reversal follows a complex path. While all nanodots seem to be well-aligned during saturation at first glance, a deeper look reveals some differences near the touching points between neighboring dots where the influence of the shape anisotropy is reduced due to touching the next dot. These differences become stronger for a reduced external magnetic state until a first dot switches into a vortex state, followed by the others, one after the other. Afterwards, these vortex states switch into negative saturation, again not simultaneously, but subsequently. All the steps in the resulting hysteresis loop (Figure 2a) correspond to switching one of the nanodots, similar to strongly extended Barkhausen jumps.

Next, the impact of a Ni matrix in which the Fe nanodots are embedded was tested. The results are depicted in Figure 3. Comparing both longitudinal hysteresis loops shows significantly less steps for the $\mathrm{Fe} / \mathrm{Ni}$ composite, indicating a more consistent reversal throughout the whole system. The snapshots of the magnetization reversal (Figure $3 b$ ) indeed show an abrupt switching of all four Fe nanodots at the same time, corresponding to the main jump in the curve, while the two smaller steps are related to the next reversal into negative saturation by two of the nanodots, followed by the others. Apparently, embedding the iron nanodots in the nickel matrix stabilizes the magnetization reversal process and makes it less arbitrary, which is an important factor for the possible technological application of magnetic nanodots-or especially, the vortex states available in them for many dimensions - in storage media.

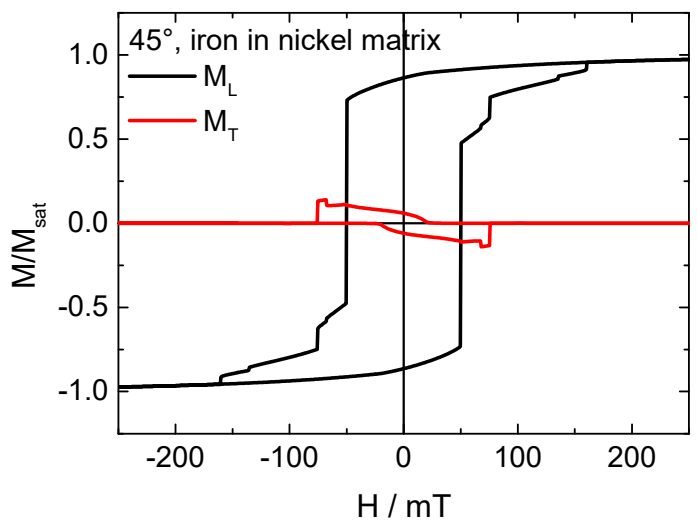

(a)
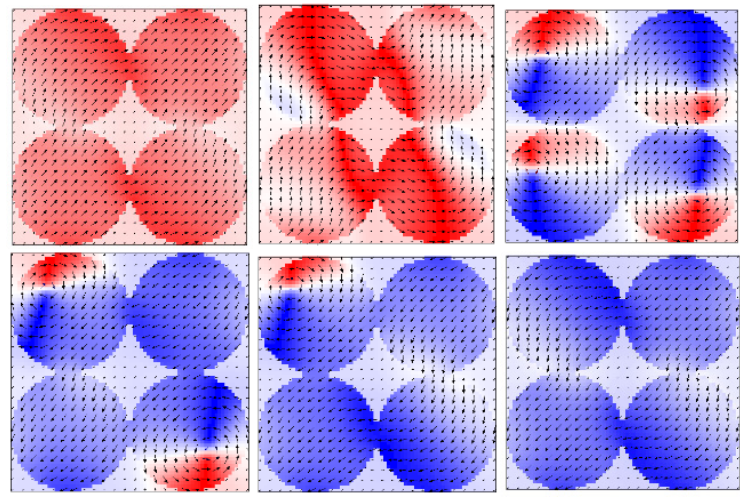

(b)

Figure 3. (a) Hysteresis loop and (b) snapshots of magnetization reversal from positive to negative saturation for a pure iron nanodot array in a nickel matrix. 
Testing the reversed matrix, Figure 4 depicts hysteresis loops and magnetization reversal snapshots from positive to negative saturation of nickel nanodots in an iron matrix. Here, the behavior is completely different. In the hysteresis loops (Figure 4a), there are strong jumps in addition to several smaller ones, combined with a large transverse magnetization component which even changes signs on either side of the loop. In the systems with Fe nanodots, this energetically unfavorable configuration of a large fraction of the magnetization being perpendicular to the external magnetic field could be avoided by building vortex states, which is not usual in materials such as nickel or permalloy with their small magneto-crystalline anisotropies [15]. Indeed, magnetization reversal snapshots show no vortices here, neither in the nickel nanodots nor in the iron antidot matrix where vortices are impeded by the shape.

Instead, domain walls are formed, preferably inside the nickel nanodots due to their small anisotropy, and only at the beginning and the end of the magnetization reversal process, these domain walls are partly located inside the Fe antidot matrix.

While in this approach of embedding $\mathrm{Ni}$ in an Fe matrix, the boundaries of the model were set next to the nanodots, our first results show that a more interesting system can possibly be created by allowing iron bridges between the nickel nanodots as well as along the borders of the system.

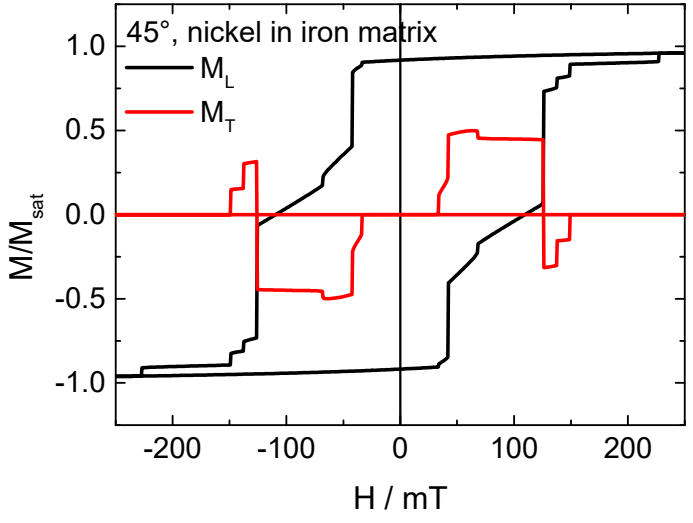

(a)

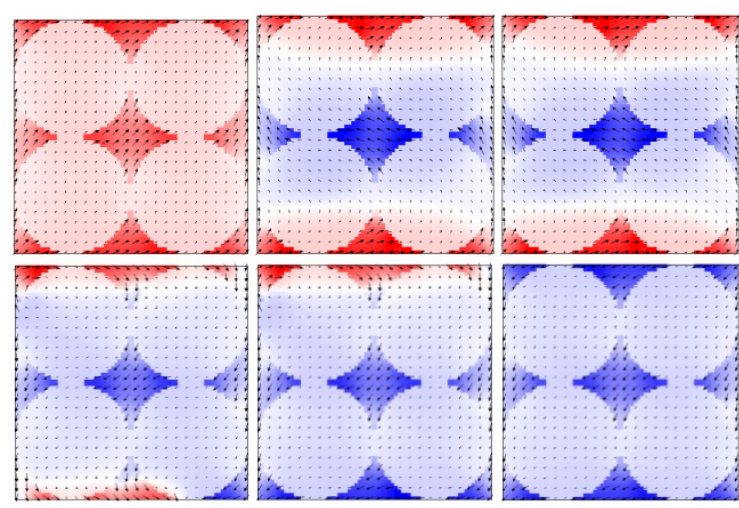

(b)

Figure 4. (a) Hysteresis loop and (b) snapshots of magnetization reversal from positive to negative saturation for a pure nickel nanodot array in an iron matrix.

\section{Conclusions}

Arrays of hard/soft magnetic material nanodots inside an antidot matrix of the opposite material were investigated by micromagnetic simulations. The results show that the magnetization reversal of Fe nanodots can be made more reliable and predictable by embedding them into a Ni matrix. Oppositely, Ni nanodots in an Fe matrix seem not to be technologically useful since magnetization reversal occurs via large domain walls through the whole system, located mostly inside the nickel nanodots.

However, more investigations on different distances between the dots are necessary to study other possible magnetic states, such as horseshoe or onion states in the Fe antidot matrix surrounding the nickel nanodots.

Supplementary Materials: The following are available online at https://www.mdpi.com/article/ 10.3390/IOCN2020-07940. 


\section{References}

1. Nogués, J.; Sort, J.; Langlais, V.; Skumryev, V.; Surinach, S.; Munoz, J.S.; Baro, M.D. Exchange bias in nanostructures. Phys. Rep. $2005,422,65$.

2. Fokin, N.; Grothe, T.; Mamun, A.; Trabelsi, M.; Klöcker, M.; Sabantina, L.; Döpke, C.; Blachowicz, T.; Hütten, A.; Ehrmann, A. Magnetic properties of electrospun magnetic nanofiber mats after stabilization and carbonization. Materials 2020, 13, 1552.

3. Wachowiak, A.; Wiebe, J.; Bode, M.; Pietzsch, O.; Morgenstern, M.; Wiesendanger, R. Direct Observation of Internal Spin Structure of Magnetic Vortex Cores. Science 2002, 298, 577-580.

4. Mejia-López, J.; Altbir, D.; Romero, A.H.; Batlle, X.; Roshchin, I.V.; Li, C.-P.; Schuller, I.K. Vortex state and effect of anisotropy in sub-100-nm magnetic nanodots. J. Appl. Phys. 2006, 100, 104319.

5. Vavassori, P.; Bovolenta, R.; Metlushko, V.; Ilic, B. Vortex rotation control in Permalloy disks with small circular voids. J. Appl. Phys. 2006, 99, 053902.

6. Guslienko, K.Y.; Aranda, G.R.; Gonzalez, J.M. Topological gauge field in nanomagnets: Spin-wave excitations over a slowly moving magnetization background. Phys. Rev. B 2010, 81, 014414.

7. Ehrmann, A.; Blachowicz, T. Systematic study of magnetization reversal in square Fe nanodots of varying dimensions in different orientations. Hyperfine Interact. 2018, 239, 48.

8. Ehrmann, A.; Blachowicz, T. Vortex and double-vortex nucleation during magnetization reversal in Fe nanodots of different dimensions. J. Magn. Magn. Mater. 2019, 475, 727-733.

9. Salaheldeen, M.; Martínez-Goyeneche, L.; Álvarez-Alonso, P.; Fernández, A. Enhancement the perpendicular magnetic anisotropy of nanopatterned hard/soft bilayer magnetic antidot arrays for spintronic applications. Nanotechnology 2020, $31,485708$.

10. Verba, R.V.; Navas, D.; Bunyaev, S.A.; Hierro-Rodriguez-A.; Guslienko, K.Y.; Ivanov, B.A.; Kakazei, G.N. Helicity of magnetic vortices and skyrmions in soft ferromagnetic nanodots and films biased by stray radial fields. Phys. Rev. B 2020, 101, 064429.

11. Negusse, E.; Williams, C.M. Magnetically induced enhanced exchange spring effect in $\mathrm{CoFe}_{2} \mathrm{O}_{4} / \mathrm{CoFe}_{2} / \mathrm{CoFe}_{2} \mathrm{O}_{4}$ films. IEEE Trans. Magn. 2020, 56, 7516705.

12. Kim, H.-Y.; Park, H.-K.; Ju, Y.-W. Fabrication of the novel $\mathrm{Fe}_{2+\alpha} \mathrm{O}_{3+\alpha}-\mathrm{CoFe}_{2} \mathrm{O}_{4}$ composite fibers and their magnetic properties. J. Korean Ceram. Soc. 2020, 57, 423-431.

13. Zhang, X.; Kan, X.C.; Wang, M.; Rao, R.; Qian, N.X.; Zheng, G.H.; Ma, Y.Q. Mechanism of enhanced magnetization in $\mathrm{CoFe}_{2} \mathrm{O}_{4} / \mathrm{La} 0.7 \mathrm{Sr}_{0.3} \mathrm{MnO}_{3}$ composites with different mass ratios. Ceram. Int. 2020, 46, 14847-14856.

14. Sudsom, D.; Juhász Junger, I.; Döpke, C.; Blachowicz, T.; Hahn, L.; Ehrmann, A. Micromagnetic Simulation of Vortex Development in Magnetic Bi-Material Bow-Tie Structures. Cond. Matter 2020, 5, 5.

15. Sudsom, D.; Blachowicz, T.; Hahn, L.; Ehrmann, A. Vortex nucleation and propagation in magnetic double-wedges and semi-squares for reliable quaternary storage systems. J. Magn. Magn. Mater. 2020, 514, 167294.

16. Donahue, M.J.; Porter, D.G. OOMMF User's Guide, Version 1.0; Interagency Report NISTIR 6376; National Institute of Standards and Technology: Gaithersburg, MD, USA, 1999.

17. Gilbert, T.L. A phenomenological theory of damping in ferromagnetic materials. IEEE Trans. Magn. 2004, 40, 3443-3449.

18. Dang Xuan, N.; Döpke, C.; Blachowicz, T.; Ehrmann, A. Magnetization reversal in hexagonal nanomagnets. Act. Phys. Pol. 2020, 137, 395-403. 